\title{
Article \\ Integral Comparison Criteria for Half-Linear Differential Equations Seen as a Perturbation
}

\author{
Zuzana Pátíková iD
}

Citation: Pátíková, Z. Integral Comparison Criteria for Half-Linear Differential Equations Seen as a Perturbation. Mathematics 2021, 9 , 502. https://doi.org/10.3390/ math9050502

Academic Editor: Ravi P. Agarwal

Received: 1 February 2021

Accepted: 24 February 2021

Published: 1 March 2021

Publisher's Note: MDPI stays neutral with regard to jurisdictional claims in published maps and institutional affiliations.

Copyright: (c) 2021 by the authors. Licensee MDPI, Basel, Switzerland. This article is an open access article distributed under the terms and conditions of the Creative Commons Attribution (CC BY) license (https:/ / creativecommons.org/licenses/by/ $4.0 /)$.
Department of Mathematics, Tomas Bata University in Zlín, Nad Stráněmi 4511, 76005 Zlín, Czech Republic; patikova@utb.cz; Tel.: +420-57-603-5005

\begin{abstract}
In this paper, we present further developed results on Hille-Wintner-type integral comparison theorems for second-order half-linear differential equations. Compared equations are seen as perturbations of a given non-oscillatory equation, which allows studying the equations on the borderline of oscillation and non-oscillation. We bring a new comparison theorem and apply it to the so-called generalized Riemann-Weber equation (also referred to as a Euler-type equation).
\end{abstract}

Keywords: half-linear differential equation; oscillation criteria; modified Riccati technique; Eulertype equation; second-order differential equation

MSC: $34 \mathrm{C} 10$

\section{Introduction}

In this paper, we continue our research on Hille-Wintner-type comparison criteria for half-linear, second-order differential equations and provide an answer to one of the open problems stated in [1]. We study the equation of the form

$$
l[x]:=\left(r(t) \Phi\left(x^{\prime}\right)\right)^{\prime}+c(t) \Phi(x)=0, \quad \Phi(x)=|x|^{p-1} \operatorname{sgn} x, \quad p>1,
$$

where $r, c$ are continuous functions and $r(t)>0$. Equation (1) can be seen as a generalization of the second-order linear Sturm-Liouville linear equation, to which it reduces for $p=2$, and it is well-known that many techniques for linear equations work effectively for half-linear equations too. Recall that one of the differences between half-linear and linear equations is well-visible in the notation-the attribute "half-linear" refers to the fact that the solution space of (1) has only one of the two linearity properties, where it is homogenous but not additive. On the other hand, classification of solutions and equations in terms of oscillation remains the same-a solution is called oscillatory if it has got infinitely many zeros tending to infinity, and non-oscillatory otherwise; and since oscillatory and non-oscillatory solutions cannot coexist, equations are classified as oscillatory or non-oscillatory according to their solutions. To refer to the most current results of the oscillation theory of (1), let us mention, for example, papers [2-6].

Because we are interested in the qualitative behavior of solutions of (1), we study it on a neighborhood of infinity, that is, on intervals of the form $t \geq t_{0}$, where $t_{0}$ is a real constant. By saying that a condition holds for large $t$, we mean that there exists such an interval-neighborhood of infinity, where the condition holds.

In our research, we focus on comparison theorems which compare two equations and their oscillatory properties. Let us consider another half-linear equation

$$
L[x]:=\left(r(t)\left(\Phi\left(x^{\prime}\right)\right)^{\prime}+C(t) \Phi(x)=0 .\right.
$$

Comparing the coefficient functions $c(t)$ and $C(t)$ (and even $r(t)$ and its counterpart $R(t)$ in general) pointwise, leads to the Sturm comparison theorems, whereas comparing 
integrals with coefficient functions aims at Hille-Wintner-type criteria. In formulation of classical Hille-Wintner criteria for half-linear equations, one distinguishes two cases, depending on the behavior of the integral $\int^{\infty} r^{1-q}(t) d t$. In case of its divergence and under the assumption that $\int^{\infty} C(t) d t<\infty$, the criterion says that if

$$
0 \leq \int_{t}^{\infty} c(s) d s \leq \int_{t}^{\infty} C(s) d s \text { for large } t
$$

and (2) is non-oscillatory, then (1) is non-oscillatory too, see [7] or [8] Section 2.3.1. If the integral $\int^{\infty} r^{1-q}(t) d t$ converges, denote $\rho(t):=\int_{t}^{\infty} r^{1-q}(s) d s$ and suppose that $c(t) \geq 0$, $C(t) \geq 0$ for large $t$. If

$$
\int_{t}^{\infty} c(s) \rho^{p}(s) d s \leq \int_{t}^{\infty} C(s) \rho^{p}(s) d s<\infty \text { for large } t,
$$

then non-oscillation of (2) implies non-oscillation of (1) (see [9] or [8] Section 2.3.1).

Inspired by these results, in paper [1] we adopted the view of the perturbation principle, which allows to refine the results on the threshold between oscillation and non-oscillation, and proved the generalized version of the Hille-Wintner criterion in the following setting. Together with (1) and (2), let us have the equation of the same form

$$
\tilde{L}[x]:=\left(r(t)\left(\Phi\left(x^{\prime}\right)\right)^{\prime}+\tilde{c}(t) \Phi(x)=0,\right.
$$

which is supposed to be non-oscillatory, and let $h$ be its positive principal solution. Equations (1) and (2) can be seen as perturbations of (3). The main result of [1] showed for the case $\int^{\infty} r^{1-q}(t) d t=\infty$ that under certain assumptions (see Theorem 1 below), the inequality

$$
0 \leq \int_{t}^{\infty}(c(s)-\tilde{c}(s)) h^{p}(s) d s \leq \int_{t}^{\infty}(C(s)-\tilde{c}(s)) h^{p}(s) d s<\infty
$$

together with non-oscillation of (2) ensure non-oscillation of (1).

As an immediate consequence, we obtained a comparison theorem, where in the place of the equation which is being perturbed, we have the half-linear Euler equation

$$
\left(\Phi\left(y^{\prime}\right)\right)^{\prime}+\frac{\gamma_{p}}{t^{p}} \Phi(y)=0
$$

Here, $\gamma_{p}=\left(\frac{p-1}{p}\right)^{p}$ is the so-called oscillation constant of (4), since it is the greatest possible constant for which the Euler equation is non-oscillatory, for larger constants at that place the equation oscillates. Its principal solution is known exactly and is equal to $t^{\frac{p-1}{p}}$. Another well-known equation that lies on the boundary between oscillation and non-oscillation is the so-called (generalized) Riemann-Weber equation (also referred to as the Euler-Weber equation or just the Euler-type equation). However, the principal solution of this equation cannot be expressed explicitly, and only its asymptotic form is known; hence, the criterion from [1] cannot be applied to it. This was the reason for mentioning the open problem in [1], whether the principal solution in the criterion can be replaced by a function, which is, in some sense, only close to it. As the technique concerning the so-called modified Riccati equation has been developed in more depth over the last few years (see, for example, [10]), we can now show that the answer is positive.

The paper is organized as follows. In the next section, we recall the Riccati technique, including the usage of the modified Riccati equation, the concept of the principal solution, technical lemmas, and remind the original theorem from [1] in its full version. In the section with the main results, we state and prove the main theorem and show some of its consequences for Riemann-Weber-type equations. The last part brings several concluding remarks. 


\section{Preliminaries}

Supposing that Equation (1) is non-oscillatory, it is a well-known fact that if $x$ is its solution, then the function $w=r \Phi\left(\frac{x^{\prime}}{x}\right)$ solves the relevant Riccati equation

$$
w^{\prime}(t)+c(t)+(p-1) r^{1-q}(t)|w(t)|^{q}=0, \quad q=\frac{p}{p-1},
$$

on some interval of the form $[T, \infty)$, and conversely, the solvability of (5) on an interval $[T, \infty)$ guarantees non-oscillation of (1). Here, we refer to the basic literature, for example, [8] (Section 1.1.4), for introduction to the theory (see also [11]).

It can be shown (as introduced by [12]) that among all non-oscillatory solutions of (5), there exists the minimal one $\tilde{w}$, for which any other solution of (5) satisfies the inequality $w(t)>\tilde{w}(t)$ for large $t$. Then, the solution of (5) given by

$$
\tilde{x}=K \exp \left\{\int^{t} r^{1-q}(s) \Phi^{-1}(\tilde{w}(s)) d s\right\},
$$

is called "principal" and it is related to the minimal solution of (5) by the formula $\tilde{w}=r \Phi\left(\tilde{x}^{\prime} / \tilde{x}\right)$. Note that $\Phi^{-1}$ is the inverse operator to $\Phi$ and $q$ is the so-called conjugate number to $p$, and $\frac{1}{p}+\frac{1}{q}=1$ holds.

The concept of the minimal solution of the Riccati equation is also known from the theory of linear differential equations, where the so-called integral characterization holds. Its possible extension to half-linear equations was studied, for example, in [13,14]. In [14], it was shown that the condition $\int^{\infty} \frac{d t}{r(t) x^{2}(t)\left|x^{\prime}(t)\right|^{p-2}}=\infty$ is under certain assumptions necessary or sufficient for $x$ to be the principal solution, but a complete "both-way" integral characterization has not been proven.

Now, let us turn our attention to the modified Riccati technique. Let $h(t)$ be a differentiable function such that $h(t) \geq 0$ and $h^{\prime}(t) \neq 0$ for large $t$, and let us use the notation

$$
R(t):=r(t) h^{2}(t)\left|h^{\prime}(t)\right|^{p-2}, \quad G(t):=r(t) h(t) \Phi\left(h^{\prime}(t)\right) .
$$

It was shown, for example, in [10] (Lemma 4) that a neigborhood of infinity solvability of (5) (and hence, also non-oscillation of (1)) is equivalent to solvability of the so-called modified Riccati equation

$$
v^{\prime}(t)+h(t) l[h](t)+(p-1) r^{1-q}(t) h^{-q}(t) H(v, G)=0,
$$

where

$$
H(v, G):=|v+G|^{q}-q \Phi^{-1}(G) v-|G|^{q} .
$$

The solution $v$ of the modified Riccati Equation (6) and the solution $w$ of the Riccati Equation (5) satisfy the relation $v=h^{p} w-G$.

The behavior of $H(v, G)$ was deeply described, for example, in [10], and we present here only those parts of its Lemma 5 and 6 , which are relevant for us.

Lemma 1. The function $H(v, G)$ has the following properties:

(i) $H(v, G) \geq 0$ with the equality if and only if $v=0$.

(ii) For every $L>0$, there exist constants $K_{1}=K_{1}(L)>0, K_{2}=K_{2}(L)>0$ such that

$$
K_{1}|G(t)|^{q-2} v^{2} \leq H(v, G) \leq K_{2}|G(t)|^{q-2} v^{2}
$$

for any $t$ and $v$ satisfying $\left|\frac{v}{G}\right| \leq L$.

The nonnegativity of solutions of the modified Riccati Equation (6) was studied in several papers. The following lemma summarizes results which are already adjusted to 
our needs and based on Lemma 4 and a part of the proof of Theorem 4 in [15] (for more resources see references therein).

Lemma 2. Let $h$ be a positive, continuously differentiable function, such that $h^{\prime}(t) \neq 0$ and $h(t) l[h](t) \geq 0$ for large $t$. Let $\int^{\infty} R^{-1}(t) d t=\infty$ and

$$
\left(\liminf _{t \rightarrow \infty}|G(t)|>0 \text { and } \limsup _{t \rightarrow \infty}|G(t)|<\infty\right) \text { or } \quad \lim _{t \rightarrow \infty}|G(t)|=\infty .
$$

Then, all proper solutions of (6) are nonnegative.

Finally, let us present the main theorem of [1]. Note that $h$ is here the principal solution, and assumption (7) is the condition appearing in its possible integral characterization.

Theorem 1. Let $\int^{\infty} r^{1-q}(t) d t=\infty$. Suppose that Equation (3) is non-oscillatory and possesses a positive principal solution $h$, such that there exists a finite limit

$$
\lim _{t \rightarrow \infty} G(t)=: L>0
$$

and

$$
\int^{\infty} R^{-1}(t) d t=\infty
$$

Further, suppose that $0 \leq \int_{t}^{\infty} C(s) d s<\infty$ and

$$
0 \leq \int_{t}^{\infty}(c(s)-\tilde{c}(s)) h^{p}(s) d s \leq \int_{t}^{\infty}(C(s)-\tilde{c}(s)) h^{p}(s) d s<\infty,
$$

all for large t. If Equation (2) is non-oscillatory, then (1) is also non-oscillatory.

\section{Main Results}

In this section, we present the main theorem and its corollaries.

Theorem 2. Suppose that there exists a positive continuously differentiable function $h(t)$ such that $h^{\prime}(t) \neq 0$ for large $t$ and the following conditions hold:

$$
\begin{gathered}
\int_{t}^{\infty} R^{-1}(s) d s=\infty, \\
h(t) L[h](t) \geq 0, \\
\int_{t}^{\infty} h(s) \tilde{L}[h](s) d s<\infty, \\
\left(\liminf _{t \rightarrow \infty}|G(t)|>0 \text { and } \limsup _{t \rightarrow \infty}|G(t)|<\infty\right) \text { or } \lim _{t \rightarrow \infty}|G(t)|=\infty,
\end{gathered}
$$

all for large $t$.

Let the inequality

$$
-\int_{t}^{\infty} h(s) \tilde{L}[h](s) d s \leq \int_{t}^{\infty}(c(s)-\tilde{c}(s)) h^{p}(s) d s \leq \int_{t}^{\infty}(C(s)-\tilde{c}(s)) h^{p}(s) d s<\infty
$$

be satisfied. Then, if Equation (2) is non-oscillatory, Equation (1) is non-oscillatory too.

Proof. Suppose that Equation (2) is non-oscillatory. Let $x$ be its solution. Then, the function $w=r \Phi\left(\frac{x^{\prime}}{x}\right)$ solves on an interval $[T, \infty)$ the relevant Riccati equation

$$
w^{\prime}(t)+C(t)+(p-1) r^{1-q}(t)|w(t)|^{q}=0
$$


and the function $v=h^{p} w-G$ solves the modified Riccati equation

$$
v^{\prime}(t)+h(t) L[h](t)+(p-1) r^{1-q}(t) h^{-q}(t) H(v, G)=0 .
$$

Because $H(v, G) \geq 0$ (see Lemma 1 ) and $h(t) L[h](t) \geq 0$ for large $t$ by (9), we observe that $v^{\prime}(t) \leq 0$ and the function $v(t)$ is non-increasing for large $t$. According to Lemma 2, the function $v(t)$ is non-negative, and there exists a non-negative finite $\operatorname{limit} \lim _{t \rightarrow \infty} v(t)$.

If $\lim _{t \rightarrow \infty}|G(t)|=\infty$, then we immediately see that $\left|\frac{v}{G}\right| \rightarrow 0$ for $t \rightarrow \infty$.

Now we show the same for the remaining case if $\liminf _{t \rightarrow \infty}|G(t)|>0$ and $\limsup _{t \rightarrow \infty}|G(t)|<\infty$. Integrating (13) over the interval $[T, t]$ yields

$$
v(T)-v(t)=\int_{T}^{t} h(s) L[h](s)+(p-1) r^{1-q}(s) h^{-q}(s) H(v(s), G(s)) d s,
$$

and hence,

$$
v(T) \geq \int_{T}^{t} h(s) L[h](s)+(p-1) r^{1-q}(s) h^{-q}(s) H(v(s), G(s)) d s .
$$

Now we have (suppressing the argument)

$$
h L[h]=h\left\{\left(r \Phi\left(h^{\prime}\right)\right)^{\prime}+\tilde{c} \Phi(h)+(C-\tilde{c}) \Phi(h)\right\}=h \tilde{L}[h]+(C-\tilde{c}) h^{p}
$$

and thanks to (10) and (12), we observe that $\int^{\infty} h(t) L[h](t) d t<\infty$. Let $t \rightarrow \infty$ in (14) imply the convergence of the integral

$$
\int^{\infty}(p-1) r^{1-q}(t) h^{-q}(t) H(v(t), G(t)) d t<\infty .
$$

With respect to our assumption —-the first part of (11) —-there exists a constant $L>0$ and $T_{1} \geq T$ such that $\left|\frac{v(t)}{G(t)}\right|<L$ for $t \geq T_{1}$. By Lemma 1 , there exists $K>0$ such that

$$
K|G(t)|^{q-2} v^{2}(t) \leq H(v(t), G(t)) \quad \text { for } \quad t \geq T_{1},
$$

which means

$$
K \frac{v^{2}(t)}{R(t)} \leq r^{1-q}(t) h^{-q}(t) H(v(t), G(t)) \quad \text { for } \quad t \geq T_{1} .
$$

Integrate the inequality over the interval $\left[T_{2}, \infty\right)$, where $T_{2} \geq T_{1}$ :

$$
K_{2} \int_{T_{2}}^{\infty} \frac{v^{2}(t)}{R(t)} d t \leq \int_{T_{2}}^{\infty} r^{1-q}(t) h^{-q}(t) H(v(t), G(t)) d t<\infty .
$$

By (8), we see that $v \rightarrow 0$ for $t \rightarrow \infty$, and hence, $v$ satisfies the integral equation

$$
v(t)=\int_{t}^{\infty} h(s) L[h](s)+(p-1) r^{1-q}(s) h^{-q}(s) H(v(s), G(s)) d s .
$$

Now let us define the following integral operator

$$
F(u)=\int_{t}^{\infty} h(s) l[h](s)+(p-1) r^{1-q}(s) h^{-q}(s) H(u(s), G(s)) d s
$$

on the set

$$
U=\{u(t), 0 \leq u(t) \leq v(t), t \in[T, \infty)\} .
$$

Our aim is to show that $F$ on $U$ fulfills such conditions that it has got a fixed point. Up to this point, first observe that

$$
H_{u}^{\prime}(u, G)=q \Phi^{-1}(|u+G|)-q \Phi^{-1}(G),
$$


and since $\Phi^{-1}$ is increasing and $u \geq 0$ on $U$, it means that $H_{u}^{\prime} \geq 0$, that is, $H$ is increasing in the first variable. Let us take functions $u_{1}(t), u_{2}(t)$ such that $0 \leq u_{1}(t) \leq u_{2}(t) \leq v(t)$, then the inequality $F\left(u_{1}\right) \leq F\left(u_{2}\right)$ holds too. To verify that the operator $F$ maps the set $U$ to itself, we consider the inequality

$$
0 \leq F(0) \leq F(u) \leq F(v) \leq v .
$$

The middle two inequalities hold on $U$ according to the previous paragraph. Since

$$
F(0)=\int_{t}^{\infty} h(s) l[h](s) d s=\int_{t}^{\infty} h(t) \tilde{L}[h](s)+(c(s)-\tilde{c}(s)) h^{p}(s) d s,
$$

the first inequality in (16) holds by the first inequality in (12). The last inequality

$$
F(v)=\int_{t}^{\infty} h(s) l[h](s)+(p-1) r^{1-q}(s) h^{-q}(s) H(v(s), G(s)) d s \leq v
$$

follows from the fact that

$$
h l[h]=h \tilde{L}[h]+(c-\tilde{c}) h^{p}, \quad h L[h]=h \tilde{L}[h]+(C-\tilde{c}) h^{p}
$$

together with (12) and (15).

Furthermore, $F(U)$ is obviously bounded on closed subintervals of $[T, \infty)$. To show that $F$ is uniformly continuous, let $u \in U$ be arbitrary, $\varepsilon>0$ and take $t_{1}, t_{2} \in[T, \infty]$ such that (without loss of generality) $t_{1}<t_{2}$. Denote $f(t)=h(t) l[h](t)+(p-1) r^{1-q}(t) h^{-q}(t) H(u(t)$, $G(t))$. We have

$$
\begin{aligned}
& \left|F(u)\left(t_{2}\right)-F(u)\left(t_{1}\right)\right| \\
& =\left|\int_{t_{2}}^{\infty} f(t) d t-\int_{t_{1}}^{\infty} f(t) d t\right|=\left|\int_{t_{1}}^{t_{2}} f(t) d t\right| \\
& \leq\left|\int_{t_{1}}^{t_{2}} h(t) l[h](t) d t\right|+\left|\int_{t_{1}}^{t_{2}}(p-1) r^{1-q}(t) h^{-q}(t) H(u(t), G(t)) d t\right| .
\end{aligned}
$$

Since both the integrals converge, there exists $\delta$ such that each of the integrals in absolute value is less than $\frac{\varepsilon}{2}$ for $\left|t_{2}-t_{1}\right|<\delta$ and

$$
\left|F(u)\left(t_{2}\right)-F(u)\left(t_{1}\right)\right|<\frac{\varepsilon}{2}+\frac{\varepsilon}{2}=\varepsilon .
$$

Hence, $F$ is uniformly continuous. Using the Schauder-Tychonov theorem, there exists a fixed point of $F$ on $U$ such that $F(u)=u$ and $u$ solves the integral equation

$$
u(t)=\int_{t}^{\infty} h(s) l[h](s)+(p-1) r^{1-q}(s) h^{-q}(s) H(u(s), G(s)) d s
$$

and also the modified Riccati Equation (6), and $w=h^{-p}(u+G)$ is a solution of the Riccati equation joined with (1). Hence, Equation (1) is non-oscillatory.

As an immediate consequence of the previous theorem, we have the following statement.

Corollary 1. Let the assumptions of Theorem 2 be satisfied. Then, the oscillation of Equation (1) implies that of (2).

Now, for the sake of clarity, recall that by $\log$ we mean the natural logarithm, $\log _{k}$ stands for an iterative logarithm, and $\log _{j}$ is a product of these functions according to the following definition:

$$
\log _{1} t=\log t, \quad \log _{k} t=\log _{k-1}(\log t), k \geq 2, \quad \log _{j} t=\Pi_{k=1}^{j} \log _{k} t .
$$


Let us consider the generalized Riemann-Weber half-linear equation with critical coefficients

$$
L_{R W}[x]:=\left(\Phi\left(x^{\prime}\right)\right)^{\prime}+\left(\frac{\gamma_{p}}{t^{p}}+\sum_{j=1}^{n} \frac{\mu_{p}}{t^{p} \log _{j}^{2} t}\right) \Phi(x)=0,
$$

where $n \in \mathbb{N}$ and $\mu_{p}=\frac{1}{2}\left(\frac{p-1}{p}\right)^{p-1}$. The consequence of Theorem 2 for the case where the non-oscillatory Equation (3), which is being perturbed, is set to be the Equation (17), reads as follows.

Corollary 2. Suppose that the condition

$$
L\left[t^{\frac{p-1}{p}} \log _{n}^{\frac{1}{p}} t\right] \geq 0
$$

holds for large $t$. If the inequality

$$
-\int_{t}^{\infty} f d s \leq \int_{t}^{\infty}(c-\tilde{c}) s^{p-1} \log _{n}(s) d s \leq \int_{t}^{\infty}(C-\tilde{c}) s^{p-1} \log _{n}(s) d s<\infty,
$$

where $f(s)$ is defined by (19) (see below) and $\tilde{c}(t)=\left(\frac{\gamma_{p}}{t^{p}}+\sum_{j=1}^{n} \frac{\mu_{p}}{t^{p} \log _{j}^{2} t}\right)$, is satisfied, and if Equation (2) is non-oscillatory, then Equation (1) is non-oscillatory too.

Proof. First, note that Equation (17) is non-oscillatory, and it has, in a certain sense, the largest possible coefficient function $\tilde{c}$, for which the non-oscillation is preserved. Indeed, equation

$$
\left(\Phi\left(x^{\prime}\right)\right)^{\prime}+\left(\frac{\gamma_{p}}{t^{p}}+\sum_{j=1}^{n-1} \frac{\mu_{p}}{t^{p} \log _{j}^{2} t}+\frac{\mu}{t^{p} \log _{n}^{2} t}\right) \Phi(x)=0
$$

is conditionally oscillatory, $\mu=\mu_{p}$ is its oscillation constant, and it is oscillatory for $\mu>\mu_{p}$ and non-oscillatory for $\mu \leq \mu_{p}$. The asymptotic formulas for the two linearly independent non-oscillatory solutions of (17) were derived in [16]. These solutions are asymptotically equivalent to the functions

$$
h_{1}(t)=t^{\frac{p-1}{p}} \log _{n}^{\frac{1}{p}} t, \quad h_{2}(t)=t^{\frac{p-1}{p}} \log _{n}^{\frac{1}{p}} t \log _{n+1}^{\frac{2}{p}} t
$$

and $h_{1}$ is asymptotically close to the principal solution.

Let us take $h(t)=h_{1}(t)$ in Theorem 2 and check the conditions. we have

Došlý in [17] showed that for $h(t)=t^{\frac{p-1}{p}} \log _{n}^{\frac{1}{p}} t$ and the operator defined in (17),

$$
h^{\prime}(t)=\frac{p-1}{p} t^{-\frac{1}{p}} \log _{n}^{\frac{1}{p}} t\left(1+\sum_{i=1}^{n} \frac{1}{(p-1) \log _{i} t}\right)
$$

and

$$
f(t):=h(t) L_{R W}[h](t)=\frac{\log _{n} t}{t \log ^{3} t}\left[\frac{2 \gamma_{p} p(2-p)}{3(p-1)^{2}}+o(1)\right] \quad \text { as } t \rightarrow \infty .
$$

Thus, (10) holds (the calculation can be found in [17] above the relation (3.9)). The condition in (9) is reduced to (18).

Next, as $t \rightarrow \infty$, we have

$$
R(t)=h^{2}(t)\left|h^{\prime}(t)\right|^{p-2}=\left(\frac{p-1}{p}\right)^{2-p} t \log _{n}(t)(1+o(1))
$$


and

$$
\int^{t} R^{-1}(s) d s=\left(\frac{p-1}{p}\right)^{2-p} \log _{n+1}(t)(1+o(1))
$$

which is divergent for $t \rightarrow \infty$, so (8) holds. Further,

$$
G(t)=h(t) \Phi\left(h^{\prime}\right)=\left(\frac{p-1}{p}\right) \log _{n}(t)(1+o(1))
$$

and it tends to infinity for $t \rightarrow \infty$, and hence, (11) is also satisfied.

In the next corollary, we apply the results to the generalized Riemann-Weber equation with $n+1$ terms in the sum as the testing Equation (2) in order to obtain a Hille-Wintnertype comparison criterion for the perturbed Riemann-Weber-type equation

$$
\left(\Phi\left(x^{\prime}\right)\right)^{\prime}+\left(\frac{\gamma_{p}}{t^{p}}+\sum_{j=1}^{n} \frac{\mu_{p}}{t^{p} \log _{j}^{2} t}+g(t)\right) \Phi(x)=0 .
$$

Corollary 3. Let the inequality

$$
-\int_{t}^{\infty} f(s) d s \leq \int_{t}^{\infty} g(s) s^{p-1} \log _{n}(s) d s \leq \frac{\mu_{p}}{\log _{n+1}(t)},
$$

where $f(t)$ is given by (19), hold. Then, Equation (20) is non-oscillatory.

Proof. We take (20) in place of (1),

$$
\left(\Phi\left(x^{\prime}\right)\right)^{\prime}+\left(\frac{\gamma_{p}}{t^{p}}+\sum_{j=1}^{n+1} \frac{\mu_{p}}{t^{p} \log _{j}^{2} t}\right) \Phi(x)=0
$$

in place of (2), (17) in place of (3), and $h=h_{1}=t^{\frac{p-1}{p}} \log _{n}^{\frac{1}{p}} t$. Observe that

$$
(c-\tilde{c})=g(t), \quad(C-\tilde{c})=\frac{\mu_{p}}{t^{p} \log _{n+1}^{2}(t)}
$$

and

$$
(C-\tilde{c}) h^{p}=\frac{\mu_{p}}{t^{p} \log _{n+1}^{2}(t)} t^{p-1} \log _{n}(t)=\frac{\mu_{p}}{t \log _{n}(t) \log _{n+1}^{2}(t)} .
$$

The integral

$$
\int_{t}^{\infty}(C-\tilde{c}) h^{p}=\int_{t}^{\infty} \frac{\mu_{p}}{s \log _{n}(s) \log _{n+1}^{2}(s)} d s=\frac{\mu_{p}}{\log _{n+1}(t)},
$$

as can be shown by the substitution $\log _{n+1}(s)=u$ and with the use of the fact that $\left(\log _{n+1}(t)\right)^{\prime}=\frac{1}{t \log _{n}(t)}$.

Finally, let us verify the condition (9). We have

$$
\begin{aligned}
h L[h] & =h L_{R W}[h]+(C-\tilde{c}) h^{p} \\
& =\frac{\log _{n} t}{t \log ^{3} t}\left[\frac{2 \gamma_{p} p(2-p)}{3(p-1)^{2}}+o(1)\right]+\frac{\mu_{p}}{t \log _{n}(t) \log _{n+1}^{2}(t)}
\end{aligned}
$$


as $t \rightarrow \infty$. Show that $\frac{\log _{n} t}{t \log ^{3} t}=o\left(\frac{1}{t \log _{n}(t) \log _{n+1}^{2}(t)}\right)$ as $t \rightarrow \infty$ :

$$
\lim _{t \rightarrow \infty} \frac{\frac{\log _{n} t}{t \log ^{3} t}}{\frac{1}{t \log _{n}(t) \log _{n+1}^{2}(t)}}=\lim _{t \rightarrow \infty} \frac{\log _{n+1}^{2}(t)}{\log ^{3} t}=\lim _{t \rightarrow \infty} \frac{\log _{n+1}^{2}(t)}{\log t} \leq \lim _{t \rightarrow \infty}\left(\frac{\log _{2}(t)}{\log ^{\varepsilon} t}\right)^{2 n},
$$

where $\varepsilon=\frac{1}{2 n}$. This limit tends to 0 as $t \rightarrow \infty$ since $\lim _{t \rightarrow \infty} \frac{\log _{2}(t)}{\log ^{\varepsilon} t}=0$ for $\varepsilon>0$ (as can be shown by the L'Hospital's rule). Hence, $h L[h] \geq 0$ for large $t$.

\section{Concluding Remarks}

(a) Let us mention that Corollary 3, as the specific application of Theorem 2 to concrete Equations (20) and (17), and the generalized Riemann-Weber equation with $n+1$ terms, brings a result which is in compliance with the Hille-Nehari-type criterion, that was proved in [17] (more on Hille-Nehari-type criteria for (20) can be found also in [18]). Its non-oscillatory part says the following. Suppose that the integral $\int^{\infty} \tilde{c}(t) t^{p-1} \log _{n} t d t$ is convergent. If

$$
\begin{aligned}
& \limsup _{t \rightarrow \infty} \log _{n+1} t \int_{t}^{\infty} \tilde{c}(s) s^{p-1} \log _{n} s d s<\mu_{p}, \\
& \liminf _{t \rightarrow \infty} \log _{n+1} t \int_{t}^{\infty} \tilde{c}(s) s^{p-1} \log _{n} s d s>-3 \mu_{p},
\end{aligned}
$$

then (20) is non-oscillatory.

(b) Let us observe that Theorem 2 can be applied also to the situation where the Euler Equation (4) is in the position of (3). We can use the exact principal solution $h=t^{\frac{p-1}{p}}$ for which

$$
\begin{gathered}
R(t)=\left(\frac{p-1}{p}\right)^{2-p} t(1+o(1)), \quad \int^{t} R^{-1}(s) d s=(1+o(1))\left(\frac{p-1}{p}\right)^{2-p} \log (t), \\
G(t)=\left(\frac{p-1}{p}\right)(1+o(1)) \text { and } h(t) L_{E}[h](t)=0
\end{gathered}
$$

as $t \rightarrow \infty$. Such a corollary was already presented in [1].

(c) Note that the perturbation $(c-\tilde{c})$ does not have to be less than $(C-\tilde{c})$ pointwise (then the Sturm comparison theorem would be sufficient) and $c$ can oscillate around $\tilde{c}$ as long as the integral inequality (12) holds. For results for Riemann-Weber-type half-linear equations with sums of periodic functions instead of constants, see [19].

(d) Finally, comment on the differences between Theorems 1 and 2.

Firstly, in Theorem 2, we do not suppose $\int^{\infty} r^{1-q}=\infty$ anywhere. Next, the main difference is the fact that $h$ is once a principal solution of (3) and once a function which is only close to that principal solution. The condition (8) is in both the theorems, and it is connected with the closeness of functions $h$ to the principal solution. The condition (9), that is, $h L[h] \geq 0$ for large $t$, does not have its counterpart in Theorem 1 , and here we have another difference between the theorems. The reason for this condition is in usage of Lemma 2. The assumption (10) is a variant on the condition $0 \leq \int{ }^{\infty} C(t) d t<\infty$ from Theorem 1. The condition (11) is in fact an extension of the assumption of the existence of a finite $\operatorname{limit}_{\lim _{t \rightarrow \infty}}|G(t)|$. We might ask whether (11) could be replaced just by $\lim \inf _{t \rightarrow \infty}|G(t)|>0$, but certainly, it can be replaced by a weaker condition of the existence of the limit such that $\lim _{t \rightarrow \infty}|G(t)|>0$. Note that the first part of (11) holds for the case where Equation (3) is the Euler Equation (4), whereas the second part holds for the case of Riemann-Weber type Equation (17). The last difference is in (12) in the very first inequality. In Theorem 1, the integral $\int_{t}^{\infty} h(s) \tilde{L}[h](s) d s$ is equal to 0 trivially, because $h$ is an exact solution of (3). 
Funding: This research received no external funding.

Institutional Review Board Statement: Not applicable.

Informed Consent Statement: Not applicable.

Data Availability Statement: Not applicable.

Conflicts of Interest: The author declares no conflict of interest.

\section{References}

1. Došlý, O.; Pátíková, Z. Hille-Wintner-type comparison criteria for half-linear, second-order differential equations. Arch. Math. 2006, 42, 185-194.

2. Došlá, Z.; Hasil, P.; Matucci, S.; Veselý, M. Euler type linear and half-linear differential equations and their non-oscillation in the critical oscillation case. J. Inequal. Appl. 2019, 189, 1-30. [CrossRef]

3. Fujimoto, K. Power comparison theorems for oscillation problems for second-order differential equations with $\mathrm{p}(\mathrm{t})$-Laplacian. Acta Math. Hungar. 2020, 162, 333-344. [CrossRef]

4. Hasil, P.; Jaroš, J.; Veselý, M. Riccati technique and oscillation constant for modified euler type half-linear equations. Publ. Math. Debrecen 2020, 97, 117-147. [CrossRef]

5. Šišoláková, J. Non-oscillation of linear and half-linear differential equations with unbounded coefficients. Math. Methods Appl. Sci. 2021, 44, 1285-1297. [CrossRef]

6. Takaŝi, K.; Manojlović, J.V. Asymptotic behavior of solutions of half-linear differential equations and generalized Karamata functions. Georgian Math. J. 2021, 28, 1-26.

7. Kusano, T.; Yosida, N. non-oscillation theorems for a class of quasilinear differential equations of second-order. Acta Math. Hungar. 1997, 76, 81-89. [CrossRef]

8. Došlý, O.; Řehák, P. Half-Linear Differential Equations. In North Holland Mathematics Studies 202; Elsevier: Amsterdam, The Netherlands, 2005.

9. Kusano, T.; Yosida, N.; Ogata, A. Strong oscillation and non-oscillation of quasilinear differential equations of second-order. Differ. Equ. Dyn. Syst. 1994, 2, 1-10.

10. Došlý, O.; Fišnarová, S. Half-linear oscillation criteria: Perturbation in term involving derivative. Nonlinear Anal. 2010, 73, 3756-3766. [CrossRef]

11. Agarval, R.P.; Grace, S.R.; O'Regan, D. Oscillation Theory for Second-Order Linear, Half-Linear, Superlinear and Sublinear Dynamic Equations; Kluwer Academic Publishers: Dordrecht, The Netherlands; Boston, MA, USA; London, UK, 2002.

12. Mirzov, J.D. Principal and nonprincipal solutions of a non-oscillatory system. Tbiliss. Gos. Univ. Inst. Prikl. Mat. Trudy 1988, 31, 100-117.

13. Fišnarová, S.; Mařík, R. Half-linear ODE and modified Riccati equation: Comparison theorems, integral characterization of principal solution. Nonlinear Anal. 2011, 74, 6427-6433. [CrossRef]

14. Došlý, O.; Elbert, Á. Integral characterization of the principal solution of half-linear, second-order differential equations. Studia Sci. Math. Hungar. 2000, 36, 455-469.

15. Došlý, O.; Fišnarová, S.; Mař́k, R. Power comparison theorems in half-linear oscillation theory. J. Math. Anal. Appl. 2013, 401, 611-619. [CrossRef]

16. Elbert, Á.; Schneider, A. Perturbations of the half-linear Euler differential equation. Results Math. 2000, 37, 56-83. [CrossRef]

17. Došlý, O. Half-linear Euler differential equation and its perturbations. Electron. J. Qual. Theory Differ. Equ. 2016, 10, 1-14.

18. Fišnarová, S.; Pátíková, Z. Perturbed generalized half-linear Riemann-Weber equation - further oscillation results. Electron. J. Qual. Theory Differ. Equ. 2017, 69, 1-12. [CrossRef]

19. Hasil, P.; Veselý, M. Oscillation and non-oscillation results for solutions of perturbed half-linear equations. Math. Methods Appl. Sci. 2018, 41, 3246-3269. [CrossRef] 